\title{
AN APPROACH TO MULTIPLE ATTRIBUTE DECISION MAKING BASED ON THE INDUCED CHOQUET INTEGRAL WITH FUZZY NUMBER INTUITIONISTIC FUZZY INFORMATION
}

\author{
Guiwu Wei' ${ }^{1}$, Rui Lin ${ }^{2}$, Xiaofei Zhao ${ }^{3}$, Hongjun Wang ${ }^{4}$ \\ School of Economics and Management, Chongqing University of Arts and Sciences, \\ Chongqing 402160, P. R. China \\ E-mails: ${ }^{1}$ weiguiwu@163.com (corresponding author); ${ }^{2}$ linrui20000@163.com; \\ 3zhao_xiaofei1980@163.com; ${ }^{4}$ wang_hongjun2008@163.com \\ Received 09 November 2011; accepted 27 June 2012
}

\begin{abstract}
In this paper, we investigate the multiple attribute decision making problems with fuzzy number intuitionistic fuzzy information. Firstly, some operational laws of fuzzy number intuitionistic fuzzy values, score function and accuracy function of fuzzy number intuitionistic fuzzy values are introduced. Then, we have developed two fuzzy number intuitionistic fuzzy Choquet integral aggregation operators: induced fuzzy number intuitionistic fuzzy choquet ordered averaging (IFNIFCOA) operator and induced fuzzy number intuitionistic fuzzy choquet ordered geometric (IFNIFCOG) operator. The prominent characteristic of the operators is that they can not only consider the importance of the elements or their ordered positions, but also reflect the correlation among the elements or their ordered positions. We have studied some desirable properties of the IFNIFCOA and IFNIFCOG operators, such as commutativity, idempotency and monotonicity, and applied the IFNIFCOA and IFNIFCOGM operators to multiple attribute decision making with fuzzy number intuitionistic fuzzy information. Finally an illustrative example has been given to show the developed method.
\end{abstract}

Keywords: Multiple attribute decision making, Fuzzy number intuitionistic fuzzy values, operational laws, induced fuzzy number intuitionistic fuzzy choquet ordered averaging operator, induced fuzzy number intuitionistic fuzzy choquet ordered geometric operator, Choquet integral.

Reference to this paper should be made as follows: Wei, G.; Lin, R.; Zhao, X.; Wang, H. 2014. An approach to multiple attribute decision making based on the induced Choquet integral with fuzzy number intuitionistic fuzzy information, Journal of Business Economics and Management 15(2): 277-298.

JEL Classification: C61.

\section{Introduction}

Multiple attribute decision making (MADM) problems are wide spread in real life decision situations. A MADM problem is to find a most desirable alternative from all feasible alternatives assessed on multiple attributes, both quantitative and qualitative 
(Kersuliene et al. 2010; Wachowicz 2010; Zavadskas et al. 2010). Atanassov (1986) introduced the concept of intuitionistic fuzzy set(IFS), which is a generalization of the concept of fuzzy set (Zadeh 1965). The intuitionistic fuzzy set has received more and more attention since its appearance. Xu and Yager (2006) developed some geometric aggregation operators, such as the intuitionistic fuzzy weighted geometric (IFWG) operator, the intuitionistic fuzzy ordered weighted geometric (IFOWG) operator, and the intuitionistic fuzzy hybrid geometric (IFHG) operator and gave an application of the IFHG operator to multiple attribute group decision making with intuitionistic fuzzy information. Xu (2007a) developed the intuitionistic fuzzy weighted averaging (IFWA) operator, the intuitionistic fuzzy ordered weighted averaging (IFOWA) operator, and the intuitionistic fuzzy hybrid aggregation (IFHA) operator. Wei (2008) utilized the maximizing deviation method for intuitionistic fuzzy multiple attribute decision making with incomplete weight information. Atanassov and Gargov (1989) introduced the concept of interval-valued intuitionistic fuzzy sets (IVIFSs) as a further generalization of that of IFSs, as well as of IVFSs. Atanassov (1994) defined some operational laws of the IVIFSs. Xu and Chen (2007) developed some arithmetic aggregation operators, such as the interval-valued intuitionistic fuzzy weighted averaging (IIFWA) operator, the interval-valued intuitionistic fuzzy ordered weighted averaging (IIFOWA) operator, and the interval-valued intuitionistic fuzzy hybrid aggregation (IIFHA) operator and gave an application of the IIFHA operator to multiple attribute group decision making with interval-valued intuitionistic fuzzy information. Xu (2007b) developed some geometric aggregation operators, such as the interval-valued intuitionistic fuzzy weighted geometric (IIFWG) operator and the interval-valued intuitionistic fuzzy geometric (IIFG) operator and gave an application of the IIFWG and IIFG operators to multiple attribute group decision making with interval-valued intuitionistic fuzzy information. Ye (2009) proposed Multicriteria fuzzy decision-making method based on a novel accuracy function under interval-valued intuitionistic fuzzy environment. Wei (2009) investigated the dynamic intuitionistic fuzzy multiple attribute decision making problems and proposed the dynamic intuitionistic fuzzy weighted geometric (DIFWG) operator and uncertain dynamic intuitionistic fuzzy weighted geometric (UDIFWG) operator to aggregate dynamic or uncertain dynamic intuitionistic fuzzy information. Wei (2010a) investigated the multiple attribute group decision making (MAGDM) problems in which both the attribute weights and the expert weights take the form of real numbers, attribute values take the form of intuitionisticfuzzy numbers or interval-valued intuitionisticfuzzy numbers and proposed two new aggregation perators: induced intuitionistic fuzzy ordered weighted geometric (I-IFOWG) operator and induced interval-valued intuitionistic fuzzy ordered weighted geometric (I-IIFOWG) operator, and studied some desirable properties of the I-IFOWG and I-IIFOWG operators, such as commutativity, idempotency and monotonicity. An I-IFOWG and IFWG (intuitionisticfuzzy weighted geometric) operators-based approach is developed to solve the MAGDM problems in which both the attribute weights and the expert weights take the form of real numbers, attribute values take the form of intuitionisticfuzzy numbers. Further, they extended the developed models and procedures based on I-IIFOWG and IIFWG (interval-valued intuitionisticfuzzy weighted geometric) operators to solve the MAGDM problems in 
which both the attribute weights and the expert weights take the form of real numbers, attribute values take the form of interval-valued intuitionisticfuzzy numbers. Li (2010) proposed Linear programming method for MADM with interval-valued intuitionistic fuzzy sets. $\mathrm{Xu}$ and Xia (2011) studied the inducedgeneralized aggregation operators under intuitionisticfuzzy environments. Choquet integral and Dempster-Shafer theory of evidence are applied to aggregate inuitionistic fuzzy information and some new types of aggregation operators are developed, including the induced generalized intuitionistic fuzzy Choquet integral operators and induced generalized intuitionistic fuzzy Dempster-Shafer operators. Then they investigated their various properties and some of their special cases. Additionally, they applied the developed operators to financial decision making under intuitionisticfuzzy environments. Some extensions in interval-valued intuitionisticfuzzy situations are also pointed out.

Liu and Yuan (2007) introduced the concept of fuzzy number intuitionistic fuzzy set (FNIFS) which fundamental characteristic of the FNIFS is that the values of its membership function and non-membership function are triangular fuzzy numbers rather than exact numbers. Wang (2008a) propose the fuzzy number intuitionistic fuzzy weighted averaging (FNIFWA) operator, fuzzy number intuitionistic fuzzy ordered weighted averaging (FNIFOWA) operator and fuzzy number intuitionistic fuzzy hybrid aggregation (FNIFHA) operator. Wang (2008b) propose some aggregation operators, including fuzzy number intuitionistic fuzzy weighted geometric (FNIFWG) operator, fuzzy number intuitionistic fuzzy ordered weighted geometric (FNIFOWG) operator and fuzzy number intuitionistic fuzzy hybrid geometric (FNIFHG) operator and develop an approach to multiple attribute group decision making (MAGDM) based on the FNIFWG and the FNIFHG operators with fuzzy number intuitionistic fuzzy information. Wei et al. (2010a) developed the induced fuzzy number intuitionistic fuzzy ordered weighted geometric (I-FIFOWG) operator and studied some desirable properties of the I-FIFOWG operators, such as commutativity, idempotency and monotonicity. An I-FIFOWG and FIFWG (fuzzy number intuitionistic fuzzy weighted geometric) operators-based approach is developed to solve the MAGDM under the fuzzy number intuitionistic fuzzy environment. Furthermore, they proposed the induced fuzzy number intuitionistic fuzzy ordered weighted averaging (I-FIFOWA) operator.

All of the existing fuzzy number intuitionistic fuzzy aggregation operators only consider situations where all the elements in the fuzzy number intuitionistic fuzzy set are independent. However, in many practical situations, the elements in the fuzzy number intuitionistic fuzzy set are usually correlative. Therefore, we need to find some new ways to deal with these situations in which the decision data in question are correlative. The Choquet integral is a very useful way of measuring the expected utility of an uncertain event, and can be utilized to depict the correlations of the decision data under consideration. Motivated by the correlation properties of the Choquet integral, in this paper we propose some fuzzy number intuitionistic fuzzy aggregation operators, whose prominent characteristic is that they can not only consider the importance of the elements or their ordered positions, but also reflect the correlations of the elements or their ordered positions. To do so, the remainder of this paper is set out as follows. In the 
next section, we introduce some basic concepts related to fuzzy number intuitionistic fuzzy sets and some operational laws of fuzzy number intuitionistic fuzzy numbers. In section 2 we have developed two induced fuzzy number intuitionistic fuzzy Choquet integral aggregation operators: induced fuzzy number intuitionistic fuzzy choquet ordered averaging (IFNIFCOA) operator and induced fuzzy number intuitionistic fuzzy choquet ordered geometric (IFNIFCOG) operator. In section 3, we have developed an approach to multiple attribute decision making based on IFNIFCOA operator and IFNIFCOG operator with fuzzy number intuitionistic fuzzy information. In section 4, an illustrative example is pointed out. In the last Section, we conclude the paper and give some remarks.

\section{Preliminaries}

Atanassov (1986) extended the fuzzy set to the IFS, shown as follows.

Definition 1. Given a fixed set $X=\left\{x_{1}, x_{2}, \cdots, x_{n}\right\}$, an intuitionistic fuzzy set (IFS) is defined as (Atanassov 1986):

$$
A=\left(\left\langle x_{i}, t_{A}\left(x_{i}\right), f_{A}\left(x_{i}\right)\right\rangle \mid x_{i} \in X\right),
$$

which assigns to each elements $x_{i}$ a membership degree $t_{A}\left(x_{i}\right)$ and a non-membership degree $f_{A}\left(x_{i}\right)$ under the condition:

$0 \leq t_{A}\left(x_{i}\right)+f_{A}\left(x_{i}\right) \leq 1$, for all $x_{i} \in X$.

Atanassov and Gargov (1989) further introduced the interval-valued intuitionistic fuzzy set (IVIFS), which is a generalization of the IFS. The fundamental characteristic of the IVIFS is that the values of its membership function and non-membership function are intervals rather than exact numbers.

Definition 2. Given a fixed set $X=\left\{x_{1}, x_{2}, \cdots, x_{n}\right\}$, then an IVIFSs $\tilde{A}$ over $X$ is an object having the form:

$$
\tilde{A}=\left\{\left\langle x_{i}, \tilde{t}_{A}\left(x_{i}\right), \tilde{f}_{A}\left(x_{i}\right)\right\rangle \mid x_{i} \in X\right\},
$$

where $\tilde{t}_{A}\left(x_{i}\right) \subset[0,1]$ and $\tilde{f}_{A}\left(x_{i}\right) \subset[0,1]$ are interval numbers, and $0 \leq \sup \tilde{t}_{A}\left(x_{i}\right)+\sup \tilde{f}_{A}\left(x_{i}\right) \leq 1, \forall x_{i} \in X$.

Liu and Yuan (2007) introduced the concept of fuzzy number intuitionistic fuzzy set (FNIFS) which fundamental characteristic of the FNIFS is that the values of its membership function and non-membership function are triangular fuzzy numbers.

Definition 3. Given a fixed set $X=\left\{x_{1}, x_{2}, \cdots, x_{n}\right\}$, an FNIFS $\tilde{A}$ over $X$ is an object having the form:

$$
\tilde{A}=\left\{\left\langle x_{i}, \tilde{t}_{A}\left(x_{i}\right), \tilde{f}_{A}\left(x_{i}\right)\right\rangle \mid x_{i} \in X\right\},
$$

where $\tilde{t}_{A}\left(x_{i}\right) \subset[0,1]$ and $\tilde{f}_{A}\left(x_{i}\right) \subset[0,1]$ are triangular fuzzy numbers, and $\tilde{t}_{A}\left(x_{i}\right)=\left(a\left(x_{i}\right), b\left(x_{i}\right), c\left(x_{i}\right)\right),: X \rightarrow[0,1], \tilde{f}_{A}(x)=\left(l\left(x_{i}\right), m\left(x_{i}\right), p\left(x_{i}\right)\right): X \rightarrow[0,1]$, $0 \leq c\left(x_{i}\right)+p\left(x_{i}\right) \leq 1, \forall x \in X$. 
For convenience, let $\tilde{t}_{A}\left(x_{i}\right)=\left(a\left(x_{i}\right), b\left(x_{i}\right), c\left(x_{i}\right)\right), \quad \tilde{f}_{A}(x)=\left(l\left(x_{i}\right), m\left(x_{i}\right), p\left(x_{i}\right)\right)$, so $\tilde{a}\left(x_{i}\right)=\left\langle\left(a\left(x_{i}\right), b\left(x_{i}\right), c\left(x_{i}\right)\right),\left(l\left(x_{i}\right), m\left(x_{i}\right), p\left(x_{i}\right)\right)\right\rangle$ and we call $\tilde{a}\left(x_{i}\right)$ an fuzzy number intuitionistic fuzzy value (FNIFV).

Definition 4. Let $\tilde{a}\left(x_{i}\right)=\left\langle\left(a\left(x_{i}\right), b\left(x_{i}\right), c\left(x_{i}\right)\right),\left(l\left(x_{i}\right), m\left(x_{i}\right), p\left(x_{i}\right)\right)\right\rangle$ and $\tilde{a}\left(x_{j}\right)=\left\langle\left(a\left(x_{j}\right), b\left(x_{j}\right), c\left(x_{j}\right)\right),\left(l\left(x_{j}\right), m\left(x_{j}\right), p\left(x_{j}\right)\right)\right\rangle$ be two FNIFVs, then

(1) $\tilde{a}\left(x_{i}\right) \oplus \tilde{a}\left(x_{j}\right)=$

$\left\langle\left(a\left(x_{i}\right)+a\left(x_{j}\right)-a\left(x_{i}\right) a\left(x_{j}\right), b\left(x_{i}\right)+b\left(x_{j}\right)-b\left(x_{i}\right) b\left(x_{j}\right), c\left(x_{i}\right)+c\left(x_{j}\right)-c\left(x_{i}\right) c\left(x_{j}\right)\right)\right.$, $\left.\left(l\left(x_{i}\right) l\left(x_{j}\right), m\left(x_{i}\right) m\left(x_{j}\right), p\left(x_{i}\right) p\left(x_{j}\right)\right)\right)$;

(2) $\tilde{a}\left(x_{i}\right) \otimes \tilde{a}\left(x_{j}\right)=\left\langle\left(a\left(x_{i}\right) a\left(x_{j}\right), b\left(x_{i}\right) b\left(x_{j}\right), c\left(x_{i}\right) c\left(x_{j}\right)\right)\right.$,

$\left.\left(l\left(x_{i}\right)+l\left(x_{j}\right)-l\left(x_{i}\right) l\left(x_{j}\right), m\left(x_{i}\right)+m\left(x_{j}\right)-m\left(x_{i}\right) m\left(x_{j}\right), p\left(x_{i}\right)+p\left(x_{j}\right)-p\left(x_{i}\right) p\left(x_{j}\right)\right)\right) ;$

(3) $\lambda \tilde{a}\left(x_{i}\right)=\left\langle\left(1-\left(1-a\left(x_{i}\right)\right)^{\lambda}, 1-\left(1-b\left(x_{i}\right)\right)^{\lambda}, 1-\left(1-c\left(x_{i}\right)\right)^{\lambda}\right)\right.$,

$\left.\left(\left(l\left(x_{i}\right)\right)^{\lambda},\left(m\left(x_{i}\right)\right)^{\lambda},\left(p\left(x_{i}\right)\right)^{\lambda}\right)\right), \lambda \geq 0$;

(4) $\left(\tilde{a}\left(x_{i}\right)\right)^{\lambda}=\left\langle\left(\left(a\left(x_{i}\right)\right)^{\lambda},\left(b\left(x_{i}\right)\right)^{\lambda},\left(c\left(x_{i}\right)\right)^{\lambda}\right)\right.$,

$\left.\left(1-\left(1-l\left(x_{i}\right)\right)^{\lambda}, 1-\left(1-m\left(x_{i}\right)\right)^{\lambda}, 1-\left(1-p\left(x_{i}\right)\right)^{\lambda}\right)\right\rangle, \lambda \geq 0$.

Definition 5. Let $\tilde{a}\left(x_{i}\right)=\left\langle\left(a\left(x_{i}\right), b\left(x_{i}\right), c\left(x_{i}\right)\right),\left(l\left(x_{i}\right), m\left(x_{i}\right), p\left(x_{i}\right)\right)\right\rangle$ be a FNIFV, a score function $S$ of a FNIFV $\tilde{a}\left(x_{i}\right)$ can be represented as follows (Wang 2008a):

$$
S\left(\tilde{a}\left(x_{i}\right)\right)=\frac{a\left(x_{i}\right)+2 b\left(x_{i}\right)+c\left(x_{i}\right)}{4}-\frac{l\left(x_{i}\right)+2 m\left(x_{i}\right)+p\left(x_{i}\right)}{4}, S\left(\tilde{a}\left(x_{i}\right)\right) \in[-1,1] .
$$

Definition 6. Let $\tilde{a}\left(x_{i}\right)=\left\langle\left(a\left(x_{i}\right), b\left(x_{i}\right), c\left(x_{i}\right)\right),\left(l\left(x_{i}\right), m\left(x_{i}\right), p\left(x_{i}\right)\right)\right\rangle$ be a FNIFV, an accuracy function $H$ of a FNIFV $\tilde{a}\left(x_{i}\right)$ can be represented as follows:

$H\left(\tilde{a}\left(x_{i}\right)\right)=\frac{\left(a\left(x_{i}\right)+2 b\left(x_{i}\right)+c\left(x_{i}\right)\right)+\left(l\left(x_{i}\right)+2 m\left(x_{i}\right)+p\left(x_{i}\right)\right)}{4}, H\left(\tilde{a}\left(x_{i}\right)\right) \in[0,1]$,

to evaluate the degree of accuracy of the FNIFV:

$\tilde{a}\left(x_{i}\right)=\left\langle\left(a\left(x_{i}\right), b\left(x_{i}\right), c\left(x_{i}\right)\right),\left(l\left(x_{i}\right), m\left(x_{i}\right), p\left(x_{i}\right)\right)\right\rangle$,

where $H\left(\tilde{a}\left(x_{i}\right)\right) \in[0,1]$. The larger the value of $H\left(\tilde{a}\left(x_{i}\right)\right)$, the more the degree of accuracy of the FNIFV $\tilde{a}\left(x_{i}\right)$ is.

Based on the score function $S$ and the accuracy function $H$, in the following, we shall give an order relation between two fuzzy number intuitionistic fuzzy values, which is defined as follows:

Definition 7. Let $\tilde{a}\left(x_{i}\right)=\left\langle\left(a\left(x_{i}\right), b\left(x_{i}\right), c\left(x_{i}\right)\right),\left(l\left(x_{i}\right), m\left(x_{i}\right), p\left(x_{i}\right)\right)\right\rangle$ and $\tilde{a}\left(x_{j}\right)=\left\langle\left(a\left(x_{j}\right), b\left(x_{j}\right), c\left(x_{j}\right)\right),\left(l\left(x_{j}\right), m\left(x_{j}\right), p\left(x_{j}\right)\right)\right\rangle$ be two FNIFVs, $s\left(\tilde{a}\left(x_{i}\right)\right)$ and $s\left(\tilde{a}\left(x_{j}\right)\right)$ be the scores of $\tilde{a}\left(x_{i}\right)$ and $\tilde{a}\left(x_{j}\right)$, respectively, and let $H\left(\tilde{a}\left(x_{i}\right)\right)$ 
and $H\left(\tilde{a}\left(x_{j}\right)\right)$ be the accuracy degrees of $\tilde{a}\left(x_{i}\right)$ and $\tilde{a}\left(x_{j}\right)$, respectively, then if $s\left(\tilde{a}\left(x_{i}\right)\right)<s\left(\tilde{a}\left(x_{j}\right)\right)$, then $\tilde{a}\left(x_{i}\right)$ is smaller than $\tilde{a}\left(x_{j}\right)$, denoted by $\tilde{a}\left(x_{i}\right)<\tilde{a}\left(x_{j}\right)$; if $s\left(\tilde{a}\left(x_{i}\right)\right)=s\left(\tilde{a}\left(x_{j}\right)\right)$, then

(1) if $H\left(\tilde{a}\left(x_{i}\right)\right)=H\left(\tilde{a}\left(x_{j}\right)\right)$, then $\tilde{a}\left(x_{i}\right)$ and $\tilde{a}\left(x_{j}\right)$ represent the same information, denoted by $\tilde{a}\left(x_{i}\right)=\tilde{a}\left(x_{j}\right)$;

(2) if $H\left(\tilde{a}\left(x_{i}\right)\right)<H\left(\tilde{a}\left(x_{j}\right)\right), \tilde{a}\left(x_{i}\right)$ is smaller than $\tilde{a}\left(x_{j}\right)$, denoted by $\tilde{a}\left(x_{i}\right)<\tilde{a}\left(x_{j}\right)$. However, the above aggregation operators with fuzzy number intuitionistic fuzzy information is based on the assumption that the attribute of decision makers are independent, which is characterized by an independence axiom (Keeney, Raiffa 1976; Wakker 1999), that is, these operators are based on the implicit assumption that attributes of decision makers are independent of one another; their effects are viewed as additive. For real decision making problems, there is always some degree of inter-dependent characteristics between attributes. Usually, there is interaction among attributes of decision makers. However, this assumption is too strong to match decision behaviors in the real world. The independence axiom generally can't be satisfied. Thus, it is necessary to consider this issue.

Let $\mu\left(x_{i}\right)(i=1,2, \cdots, n)$ be the weight of the elements $x_{i} \in X(i=1,2, \cdots, n)$, where $\mu$ is a fuzzy measure, defined as follows:

Definition 8 (Wang, Klir 1992). A fuzzy measure $\mu$ on the set $X$ is a set function $\mu: \theta(x) \rightarrow[0,1]$ satisfying the following axioms:

(1) $\mu(\phi)=0, \mu(X)=1$;

(2) $A \subseteq B$ implies $\mu(A) \leq \mu(B)$, for all $A, B \subseteq X$;

(3) $\mu(A \cup B)=\mu(A)+\mu(B)+\rho \mu(A) \mu(B)$, for all $A, B \subseteq X$ and $A \cap B=\phi$, where $\rho \in(-1, \infty)$.

Especially, if $\rho=0$, then the condition (3) reduces to the axiom of additive measure: $\mu(A \cup B)=\mu(A)+\mu(B)$, for all $A, B \subseteq X$ and $A \cap B=\phi$.

If all the elements in $X$ are independent, and we have $\mu(A)=\sum_{x_{i} \in A} \mu\left(\left\{x_{i}\right\}\right)$, for all $A \subseteq X$.

Definition 9 (Grabisch et al., 2000). Let $f$ be a positive real-valued function on $X$, and $\mu$ be a fuzzy measure on $X$. The discrete Choquet integral of $f$ with respective to $\mu$ is defined by:

$$
C_{\mu}(f)=\sum_{i=1}^{n} f_{\sigma(i)}\left[\mu\left(A_{\sigma(i)}\right)-\mu\left(A_{\sigma(i-1)}\right)\right],
$$

where $(\sigma(1), \sigma(2), \cdots, \sigma(n))$ is a permutation of $(1,2, \cdots, n)$, such that $f_{\sigma(i-1)} \geq f_{\sigma(i)}$ for all $j=2, \cdots, n, A_{\sigma(k)}=\left\{x_{\sigma(j)} \mid j \leq k\right\}$, for $k \geq 1$, and $A_{\sigma(0)}=\phi$. 
It is seen that the discrete Choquet integral is a linear expression up to a reordering of the elements.

Definition 10 (Grabisch et al. 2000). Let $f$ be a positive real-valued function on $X$ and $m$ be a fuzzy measure on $X$. The induced Choquet ordered averaging operator of dimension $n$ is a function I-COA $:\left(R^{+} \times R^{+}\right) \rightarrow R^{+}$, which is defined to aggregate the set of second argument of a list of 2 -tuples $\left(\left\langle u_{1}, f_{1}\right\rangle,\left\langle u_{2}, f_{2}\right\rangle, \cdots,\left\langle u_{n}, f_{n}\right\rangle\right)$ according to the following expression:

$$
\begin{aligned}
& {\mathrm{I}-\mathrm{COA}_{m}}\left(\left\langle u_{1}, f_{1}\right\rangle,\left\langle u_{2}, f_{2}\right\rangle, \cdots,\left\langle u_{n}, f_{n}\right\rangle\right)= \\
& \sum_{j=1}^{n} f_{\sigma(j)}\left[m\left(A_{\sigma(j)}\right)-m\left(A_{\sigma(j-1)}\right)\right],
\end{aligned}
$$

where $(\sigma(1), \sigma(2), \cdots, \sigma(n))$ is a permutation of $(1,2, \cdots, n)$, such that $u_{\sigma(i-1)} \geq u_{\sigma(i)}$ for all $j=2, \cdots, n$, i.e., $\left\langle u_{\sigma(j)}, f_{\sigma(j)}\right\rangle$ is the 2-tuple with $u_{\sigma(j)}$ the $j$ th largest value in the $\operatorname{set}\left(u_{1}, u_{2}, \cdots, u_{n}\right), A_{\sigma(k)}=\left\{x_{\sigma(j)} \mid j \leq k\right\}$, for $k \geq 1$, and $A_{\sigma(0)}=\phi$.

\section{Some induced aggregating operators based on the Choquet integral with fuzzy number intuitionistic fuzzy information}

Wang (2008a) propose the fuzzy number intuitionistic fuzzy weighted averaging (FNIFWA) operator and fuzzy number intuitionistic fuzzy ordered weighted averaging (FNIFOWA) operator.

Definition 11. Let $\tilde{a}\left(x_{i}\right)=\left\langle\left(a\left(x_{i}\right), b\left(x_{i}\right), c\left(x_{i}\right)\right),\left(l\left(x_{i}\right), m\left(x_{i}\right), p\left(x_{i}\right)\right)\right\rangle(i=1,2, \cdots, n)$ be a collection of FNIFVs, and let FNIFWA: $Q^{n} \rightarrow Q$, if

$$
\begin{aligned}
& \text { FNIFWA }_{\omega}\left(\tilde{a}\left(x_{1}\right), \tilde{a}\left(x_{2}\right), \cdots, \tilde{a}\left(x_{n}\right)\right)= \\
& \underset{i=1}{\oplus}\left(\omega_{i} \tilde{a}\left(x_{i}\right)\right)= \\
& \left\langle\left(1-\prod_{i=1}^{n}\left(1-a\left(x_{i}\right)\right)^{\omega_{i}}, 1-\prod_{i=1}^{n}\left(1-b\left(x_{i}\right)\right)^{\omega_{i}}, 1-\prod_{i=1}^{n}\left(1-c\left(x_{i}\right)\right)^{\omega_{i}}\right)\right. \\
& \left.\left(\prod_{i=1}^{n} l\left(x_{i}\right)^{\omega_{i}}, \prod_{i=1}^{n} m\left(x_{i}\right)^{\omega_{i}}, \prod_{i=1}^{n} p\left(x_{i}\right)^{\omega_{i}}\right)\right\rangle,
\end{aligned}
$$

where $\omega=\left(\omega_{1}, \omega_{2}, \cdots, \omega_{n}\right)^{T}$ be the weight vector of $\tilde{a}\left(x_{i}\right)(i=1,2, \cdots, n)$, and $\omega_{j}>0$, $\sum_{i=1}^{n} \omega_{i}=1$, then FNIFWA is called the fuzzy number intuitionistic fuzzy weighted averaging (FNIFWA) operator.

Example 1. Assume $\omega=(0.2,0.1,0.3,0.4), \tilde{a}_{1}=\langle(0.1,0.2,0.3),(0.5,0.6,0.7)\rangle$, $\tilde{a}_{2}=\langle(0.4,0.5,0.6),(0.3,0.3,0.4)\rangle, \tilde{a}_{3}=\langle(0.4,0.4,0.5),(0.4,0.4,0.5)\rangle$, and $\tilde{a}_{4}=\langle(0.3,0.4,0.5),(0.3,0.4,0.4)\rangle$, then 
$\operatorname{FNIFWA}_{\omega}\left(\tilde{a}_{1}, \tilde{a}_{2}, \tilde{a}_{3}, \tilde{a}_{4}\right)=$

$\left\langle\left(1-(1-0.1)^{0.2} \times(1-0.4)^{0.1} \times(1-0.4)^{0.3} \times(1-0.3)^{0.4} \mathrm{~J}\right.\right.$

$1-(1-0.2)^{0.2} \times(1-0.5)^{0.1} \times(1-0.4)^{0.3} \times(1-0.4)^{0.4} \mathrm{~J}$

$\left.1-(1-0.3)^{0.2} \times(1-0.6)^{0.1} \times(1-0.5)^{0.3} \times(1-0.5)^{0.4}\right)$,

$\left(0.5^{0.2} \times 0.3^{0.1} \times 0.4^{0.3} \times 0.3^{0.4}\right.$,

$0.6^{0.2} \times 0.3^{0.1} \times 0.4^{0.3} \times 0.4^{0.4}$,

$\left.\left.0.7^{0.2} \times 0.4^{0.1} \times 0.5^{0.3} \times 0.4^{0.4}\right)\right\rangle=$

$\langle(0.308,0.376,0.477),(0.362,0.421,0.478)\rangle$.

Definition 12. Let $\tilde{a}\left(x_{i}\right)=\left\langle\left(a\left(x_{i}\right), b\left(x_{i}\right), c\left(x_{i}\right)\right),\left(l\left(x_{i}\right), m\left(x_{i}\right), p\left(x_{i}\right)\right)\right\rangle(i=1,2, \cdots, n)$ be a collection of FNIFVs, An fuzzy number intuitionistic fuzzy ordered weighted averaging (FNIFOWA) operator of dimension $n$ is a mapping FNIFOWA: $Q^{n} \rightarrow Q$, that has an associated weight vector $w=\left(w_{1}, w_{2}, \cdots, w_{n}\right)^{T}$ such that $w_{j}>0$ and $\sum_{j=1}^{n} w_{j}=1$.
Furthermore:

$\operatorname{FNIFOWA}_{w}\left(\tilde{a}\left(x_{1}\right), \tilde{a}\left(x_{2}\right), \cdots, \tilde{a}\left(x_{n}\right)\right)=$

$$
\begin{aligned}
& \underset{i=1}{\oplus}\left(\omega_{i} \tilde{a}\left(x_{\sigma(i)}\right)\right)= \\
& \left\langle\left(1-\prod_{i=1}^{n}\left(1-a\left(x_{\sigma(i)}\right)\right)^{w_{i}}, 1-\prod_{i=1}^{n}\left(1-b\left(x_{\sigma(i)}\right)\right)^{w_{i}}, 1-\prod_{i=1}^{n}\left(1-c\left(x_{\sigma(i)}\right)\right)^{w_{i}}\right)\right. \\
& \left.\left(\prod_{i=1}^{n} l\left(x_{\sigma(i)}\right)^{w_{i}}, \prod_{i=1}^{n} m\left(x_{\sigma(i)}\right)^{w_{i}}, \prod_{i=1}^{n} p\left(x_{\sigma(i)}\right)^{w_{i}}\right)\right\rangle,
\end{aligned}
$$

where $(\sigma(1), \sigma(2), \cdots, \sigma(n))$ is a permutation of $(1,2, \cdots, n)$, such that $\tilde{a}\left(x_{\sigma(i-1)}\right) \geq \tilde{a}\left(x_{\sigma(i)}\right)$ for all $i=2, \cdots, n$.

Example 2. Let $\tilde{a}_{1}=\langle(0.3,0.4,0.5),(0.2,0.3,0.4)\rangle, \tilde{a}_{2}=\langle(0.3,0.3,0.3),(0.4,0.5,0.6)\rangle$, $\tilde{a}_{3}=\langle(0.5,0.5,0.5),(0.3,0.3,0.3)\rangle$, and $\tilde{a}_{4}=\langle(0.1,0.2,0.2),(0.6,0.7,0.8)\rangle$ be four FNIFVs, by (4), we calculate the scores of $\tilde{a}_{j}(j=1,2,3,4)$ :

$$
\begin{aligned}
& S\left(\tilde{a}_{1}\right)=0.1, S\left(\tilde{a}_{2}\right)=-0.2, \\
& S\left(\tilde{a}_{3}\right)=0.2, S\left(\tilde{a}_{4}\right)=-0.53 .
\end{aligned}
$$

Since

$$
S\left(\tilde{a}_{3}\right)>S\left(\tilde{a}_{1}\right)>S\left(\tilde{a}_{2}\right)>S\left(\tilde{a}_{4}\right),
$$

thus

$$
\begin{aligned}
& \tilde{a}_{\sigma(1)}=\langle(0.5,0.5,0.5),(0.3,0.3,0.3)\rangle, \tilde{a}_{\sigma(2)}=\langle(0.3,0.4,0.5),(0.2,0.3,0.4)\rangle, \\
& \tilde{a}_{\sigma(3)}=\langle(0.3,0.3,0.3),(0.4,0.5,0.6)\rangle, \tilde{a}_{\sigma(4)}=\langle(0.1,0.2,0.2),(0.6,0.7,0.8)\rangle .
\end{aligned}
$$


Suppose that $w=(0.2,0.3,0.4,0.1)$ is the weighting vector of the FNIFOWA operator. Then, by (7), it follows that:

$\operatorname{FNIFOWA}_{w}\left(\tilde{a}_{1}, \tilde{a}_{2}, \tilde{a}_{3}, \tilde{a}_{4}\right)=$

$\left\langle\left(1-(1-0.5)^{0.2} \times(1-0.3)^{0.3} \times(1-0.3)^{0.4} \times(1-0.1)^{0.1} \mathrm{~J}=\right.\right.$

$1-(1-0.5)^{0.2} \times(1-0.4)^{0.3} \times(1-0.3)^{0.4} \times(1-0.2)^{0.1} \mathrm{~J}$

$\left.1-(1-0.5)^{0.2} \times(1-0.5)^{0.3} \times(1-0.3)^{0.4} \times(1-0.2)^{0.1}\right)$,

$\left(0.3^{0.2} \times 0.2^{0.3} \times 0.4^{0.4} \times 0.6^{0.1}\right.$,

$0.3^{0.2} \times 0.3^{0.3} \times 0.5^{0.4} \times 0.7^{0.1}$

$\left.\left.0.3^{0.2} \times 0.4^{0.3} \times 0.6^{0.4} \times 0.8^{0.1}\right)\right\rangle=$

$\langle(0.329,0.367,0.400),(0.319,0.401,0.476)\rangle$.

Based on Definition 10, in what follows, we shall develop the induced fuzzy number intuitionistic fuzzy choquet ordered averaging (IFNIFCOA) operator based on the wellknown Choquet integral (Choquet 1953).

Definition 13. Let $X\left(x_{1}, x_{2}, \cdots, x_{n}\right)$ be a finite set, and $\mu$ be a fuzzy measure on $X$, and $\tilde{a}\left(x_{i}\right)=\left\langle\left(a\left(x_{i}\right), b\left(x_{i}\right), c\left(x_{i}\right)\right),\left(l\left(x_{i}\right), m\left(x_{i}\right), p\left(x_{i}\right)\right)\right\rangle \quad(i=1,2, \cdots, n)$ be a collection of FNIFVs on $X$, and $\mu$ be a fuzzy measure on $X$. An induced fuzzy number intuitionistic fuzzy choquet ordered averaging (IFNIFCOA) operator of dimension $n$ is a function IFNIFCOA: $Q^{n} \rightarrow Q$, which is defined to aggregate the set of second arguments of a collection of 2-tuples $\left(\left\langle u_{1}, \tilde{a}\left(x_{1}\right)\right\rangle,\left\langle u_{2}, \tilde{a}\left(x_{2}\right)\right\rangle, \cdots,\left\langle u_{n}, \tilde{a}\left(x_{n}\right)\right\rangle\right)$ according to the following expression:

$$
\begin{aligned}
& \operatorname{IFNIFCOA}_{\mu}\left(\left\langle u_{1}, \tilde{a}\left(x_{1}\right)\right\rangle,\left\langle u_{2}, \tilde{a}\left(x_{2}\right)\right\rangle, \cdots,\left\langle u_{n}, \tilde{a}\left(x_{n}\right)\right\rangle\right)= \\
& \bigoplus_{j=1}^{n}\left(\left(\mu\left(A_{\sigma(j)}\right)-\mu\left(A_{\sigma(j-1)}\right)\right) \tilde{a}\left(x_{j}\right)\right),
\end{aligned}
$$

where $u_{i}$ in 2-tuple $\left\langle u_{i}, \tilde{a}\left(x_{i}\right)\right\rangle$ is referred to as the order-inducing variable and $\tilde{a}\left(x_{i}\right)$ as the argument variable, $(\sigma(1), \sigma(2), \cdots, \sigma(n))$ is a permutation of $(1,2, \cdots, n)$, such that $u_{\sigma(j-1)} \geq u_{\sigma(j)}$ for all $j=2, \cdots, n, A_{(i)}=\left\{x_{(1)}, x_{(2)}, \cdots, x_{(i)}\right\}$ when $i \geq 1$ and $A_{\sigma(0)}=\phi$.

With the operation of fuzzy number intuitionistic fuzzy numbers, the IFNIFCOA operator can be transformed into the following from by induction on $n$ :

$\operatorname{IFNIFCOA}_{\mu}\left(\left\langle u_{1}, \tilde{a}\left(x_{1}\right)\right\rangle,\left\langle u_{2}, \tilde{a}\left(x_{2}\right)\right\rangle, \cdots,\left\langle u_{n}, \tilde{a}\left(x_{n}\right)\right\rangle\right)=$

$$
\begin{aligned}
& \bigoplus_{j=1}^{n}\left(\left(\mu\left(A_{\sigma(j)}\right)-\mu\left(A_{\sigma(j-1)}\right)\right) \tilde{a}\left(x_{j}\right)\right)= \\
& \left\langle\left(1-\prod_{i=1}^{n}\left(1-a\left(x_{\sigma(i)}\right)\right)^{\mu\left(A_{\sigma(i)}\right)-\mu\left(A_{\sigma(i-1)}\right)}, 1-\prod_{i=1}^{n}\left(1-a\left(x_{\sigma(i)}\right)\right)^{\mu\left(A_{\sigma(i)}\right)-\mu\left(A_{\sigma(i-1)}\right)}, 1-\prod_{i=1}^{n}\left(1-a\left(x_{\sigma(i)}\right)\right)^{\mu\left(A_{\sigma(i)}\right)-\mu\left(A_{\sigma(i-1)}\right)}\right),\right. \\
& \left.\left(\prod_{j=1}^{n}\left(l\left(x_{\sigma(i)}\right)\right)^{\mu\left(A_{\sigma(i)}\right)-\mu\left(A_{\sigma(i-1)}\right)}, \prod_{j=1}^{n}\left(m\left(x_{\sigma(i)}\right)\right)^{\mu\left(A_{\sigma(i)}\right)-\mu\left(A_{\sigma(i-1)}\right)}, \prod_{j=1}^{n}\left(p\left(x_{\sigma(i)}\right)\right)^{\mu\left(A_{\sigma(i)}\right)-\mu\left(A_{\sigma(i-1)}\right)}\right)\right\rangle,
\end{aligned}
$$


whose aggregated value is also a fuzzy number intuitionistic fuzzy number. Especially, if $\mu\left(\left\{x_{\sigma(j)}\right\}\right)=\mu\left(A_{\sigma(j)}\right)-\mu\left(A_{\sigma(j-1)}\right), i=1,2, \cdots, n$, then IFNIFCOA operator reduce to IFNIF WA operator. If $\mu(A)=\sum_{x_{j} \in A} \mu\left(\left\{x_{j}\right\}\right)$, for all $A \subseteq X$, where $|A|$ is the number of the elements in the set $A$, then $x_{j} \in A=\mu\left(A_{\sigma(j)}\right)-\mu\left(A_{\sigma(j-1)}\right), i=1,2, \cdots, n$, where $w=\left(w_{1}, w_{2}, \cdots, w_{n}\right)^{T}, w_{j} \geq 0, i=1,2, \cdots, n$, and $\sum_{j=1}^{n} w_{j}=1$, then, IFNIFCOA
operator reduce to FNIFOWA operator. It's easy to prove that the IFNIFCOA operator has the following properties.

Theorem 1 (Commutativity).

$\operatorname{IFNIFCOA}_{\mu}\left(\tilde{a}\left(x_{1}\right), \tilde{a}\left(x_{2}\right), \cdots, \tilde{a}\left(x_{n}\right)\right)=\operatorname{IFNIFCOA}_{\mu}\left(\tilde{a}^{\prime}\left(x_{1}\right), \tilde{a}^{\prime}\left(x_{2}\right), \cdots, \tilde{a}^{\prime}\left(x_{n}\right)\right)$, where $\left(\tilde{a}^{\prime}\left(x_{1}\right), \tilde{a}^{\prime}\left(x_{2}\right), \cdots, \tilde{a}^{\prime}\left(x_{n}\right)\right)$ is any permutation of $\left(\tilde{a}\left(x_{1}\right), \tilde{a}\left(x_{2}\right), \cdots, \tilde{a}\left(x_{n}\right)\right)$.

Theorem 2 (Idempotency). If $\tilde{a}\left(x_{j}\right)=\tilde{a}(x)$ for all $j$, then:

$\operatorname{IFNIFCOA}_{\mu}\left(\tilde{a}\left(x_{1}\right), \tilde{a}\left(x_{2}\right), \cdots, \tilde{a}\left(x_{n}\right)\right)=\tilde{a}(x)$

Theorem 3 (Monotonicity). If $\tilde{a}\left(x_{j}\right) \leq \tilde{a}^{\prime}\left(x_{j}\right)$ for all $j$, then:

$\operatorname{IFNIFCOA}_{\mu}\left(\tilde{a}\left(x_{1}\right), \tilde{a}\left(x_{2}\right), \cdots, \tilde{a}\left(x_{n}\right)\right) \leq$

$\operatorname{IFNIFCOA}_{\mu}\left(\tilde{a}^{\prime}\left(x_{1}\right), \tilde{a}^{\prime}\left(x_{2}\right), \cdots, \tilde{a}^{\prime}\left(x_{n}\right)\right)$.

Example 3. Assume we have four FNIFOWA pairs $\left\langle u_{j}, \tilde{a}\left(x_{j}\right)\right\rangle$ given:

$$
\begin{aligned}
& \left\langle u_{1}, \tilde{a}\left(x_{1}\right)\right\rangle=\langle 12,((0.3,0.4,0.5),(0.1,0.2,0.3))\rangle, \\
& \left\langle u_{2}, \tilde{a}\left(x_{2}\right)\right\rangle=\langle 15,((0.4,0.5,0.5),(0.1,0.1,0.2))\rangle, \\
& \left\langle u_{3}, \tilde{a}\left(x_{3}\right)\right\rangle=\langle 10,((0.2,0.3,0.3),(0.3,0.4,0.5))\rangle, \\
& \left\langle u_{4}, \tilde{a}\left(x_{4}\right)\right\rangle=\langle 8,((0.2,0.3,0.4),(0.4,0.5,0.6))\rangle .
\end{aligned}
$$

That we desire to aggregate using the weighting vector $w=(0.2,0.4,0.1,0.3)$. Performing the ordering the FNIFOWA pairs with respect to the first component, we get:

$$
\begin{aligned}
& \left\langle u_{\sigma(1)}, \tilde{a}\left(x_{\sigma(1)}\right)\right\rangle=\langle 15,((0.4,0.5,0.5),(0.1,0.1,0.2))\rangle, \\
& \left\langle u_{\sigma(2)}, \tilde{a}\left(x_{\sigma(2)}\right)\right\rangle=\langle 12,((0.3,0.4,0.5),(0.1,0.2,0.3))\rangle, \\
& \left\langle u_{\sigma(3)}, \tilde{a}\left(x_{\sigma(3)}\right)\right\rangle=\langle 10,((0.2,0.3,0.3),(0.3,0.4,0.5))\rangle, \\
& \left\langle u_{\sigma(4)}, \tilde{a}\left(x_{\sigma(4)}\right)\right\rangle=\langle 8,((0.2,0.3,0.4),(0.4,0.5,0.6))\rangle .
\end{aligned}
$$

This ordering includes the ordered fuzzy number intuitionistic fuzzy numbers:

$$
\begin{aligned}
& \tilde{a}\left(x_{\sigma(1)}\right)=\langle(0.4,0.5,0.5),(0.1,0.1,0.2)\rangle, \\
& \tilde{a}\left(x_{\sigma(2)}\right)=\langle(0.3,0.4,0.5),(0.1,0.2,0.3)\rangle,
\end{aligned}
$$




$$
\begin{aligned}
& \tilde{a}\left(x_{\sigma(3)}\right)=\langle(0.2,0.3,0.3),(0.3,0.4,0.5)\rangle, \\
& \tilde{a}\left(x_{\sigma(4)}\right)=\langle(0.2,0.3,0.4),(0.4,0.5,0.6)\rangle .
\end{aligned}
$$

Suppose the fuzzy measure of attribute of $\tilde{a}_{i}(i=1,2,3,4)$ and attribute sets of $\tilde{a}_{i}(i=1,2,3,4)$ as follows:

$\mu(\phi)=0, \mu\left(\tilde{a}_{1}\right)=0.40, \mu\left(\tilde{a}_{2}\right)=0.25, \mu\left(\tilde{a}_{3}\right)=0.38, \mu\left(\tilde{a}_{4}\right)=0.25$,

$\mu\left(\tilde{a}_{1}, \tilde{a}_{2}\right)=0.56, \mu\left(\tilde{a}_{1}, \tilde{a}_{3}\right)=0.65, \mu\left(\tilde{a}_{1}, \tilde{a}_{4}\right)=0.50, \mu\left(\tilde{a}_{2}, \tilde{a}_{3}\right)=0.45$,

$\mu\left(\tilde{a}_{2}, \tilde{a}_{4}\right)=0.39, \mu\left(\tilde{a}_{3}, \tilde{a}_{4}\right)=0.40, \mu\left(\tilde{a}_{1}, \tilde{a}_{2}, \tilde{a}_{3}\right)=0.80, \mu\left(\tilde{a}_{1}, \tilde{a}_{2}, \tilde{a}_{4}\right)=0.75$,

$\mu\left(\tilde{a}_{1}, \tilde{a}_{3}, \tilde{a}_{4}\right)=0.72, \mu\left(\tilde{a}_{2}, \tilde{a}_{3}, \tilde{a}_{4}\right)=0.60, \mu\left(\tilde{a}_{1}, \tilde{a}_{2}, \tilde{a}_{3}, \tilde{a}_{4}\right)=1.00$.

Then, by (10), it follows that:

$\operatorname{IFNIFCOA}_{\mu}\left(\left\langle u_{1}, \tilde{a}\left(x_{1}\right)\right\rangle,\left\langle u_{2}, \tilde{a}\left(x_{2}\right)\right\rangle,\left\langle u_{3}, \tilde{a}\left(x_{3}\right)\right\rangle,\left\langle u_{4}, \tilde{a}\left(x_{4}\right)\right\rangle\right)=$

$\left\langle\left(1-(1-0.4)^{0.25} \times(1-0.3)^{0.56-0.25} \times(1-0.2)^{0.80-0.56} \times(1-0.1)^{1-0.80} \mathrm{~J}\right.\right.$

$1-(1-0.5)^{0.25} \times(1-0.4)^{0.56-0.25} \times(1-0.3)^{0.80-0.56} \times(1-0.2)^{1-0.80} \mathrm{~J}$

$\left.1-(1-0.5)^{0.25} \times(1-0.5)^{0.56-0.25} \times(1-0.3)^{0.80-0.56} \times(1-0.3)^{1-0.80}\right)$,

$\left(0.1^{0.25} \times 0.1^{0.56-0.25} \times 0.3^{0.80-0.56} \times 0.3^{1-0.80}\right.$,

$0.1^{0.25} \times 0.2^{0.56-0.25} \times 0.4^{0.80-0.56} \times 0.4^{1-0.80}$,

$\left.\left.0.2^{0.25} \times 0.3^{0.56-0.25} \times 0.5^{0.80-0.56} \times 0.5^{1-0.80}\right)\right\rangle=$

$\langle(0.269,0.370,0.420),(0.162,0.228,0.339)\rangle$.

Wang (2008b) propose the fuzzy number intuitionistic fuzzy weighted geometric (FNIFWG) operator and fuzzy number intuitionistic fuzzy ordered weighted geometric (FNIFOWG) operator.

Definition 14. Let $\tilde{a}\left(x_{i}\right)=\left\langle\left(a\left(x_{i}\right), b\left(x_{i}\right), c\left(x_{i}\right)\right),\left(l\left(x_{i}\right), m\left(x_{i}\right), p\left(x_{i}\right)\right)\right\rangle(i=1,2, \cdots, n)$ be a collection of FNIFVs, and let FNIFWG: $Q^{n} \rightarrow Q$ if:

$$
\begin{aligned}
& \text { FNIFWG }_{\omega}\left(\tilde{a}\left(x_{1}\right), \tilde{a}\left(x_{2}\right), \cdots, \tilde{a}\left(x_{n}\right)\right)=\prod_{i=1}^{n} \tilde{a}\left(x_{i}\right)^{\omega_{i}}= \\
& \left\langle\left(\prod_{i=1}^{n} a\left(x_{i}\right)^{\omega_{i}}, \prod_{i=1}^{n} b\left(x_{i}\right)^{\omega_{i}}, \prod_{i=1}^{n} c\left(x_{i}\right)^{\omega_{i}}\right),\right. \\
& \left.\left(1-\prod_{i=1}^{n}\left(1-l\left(x_{i}\right)\right)^{\omega_{i}}, 1-\prod_{i=1}^{n}\left(1-m\left(x_{i}\right)\right)^{\omega_{i}}, 1-\prod_{i=1}^{n}\left(1-p\left(x_{i}\right)\right)^{\omega_{i}}\right)\right\rangle,
\end{aligned}
$$

where $\omega=\left(\omega_{1}, \omega_{2}, \cdots, \omega_{n}\right)^{T}$ be the weight vector of $\tilde{a}\left(x_{i}\right)(i=1,2, \cdots, n)$, and $\omega_{i}>0$, $\sum_{i=1}^{n} \omega_{i}=1$, then FIFWG is called the fuzzy number intuitionistic fuzzy weighted geometric (FIFWG) operator. 
Example 4. Assume $\omega=(0.2,0.1,0.3,0.4), \tilde{a}_{1}=\langle(0.1,0.2,0.3),(0.5,0.6,0.7)\rangle$, $\tilde{a}_{2}=\langle(0.4,0.5,0.6),(0.3,0.3,0.4)\rangle, \tilde{a}_{3}=\langle(0.4,0.4,0.5),(0.4,0.4,0.5)\rangle$, and $\tilde{a}_{4}=\langle(0.3,0.4,0.5),(0.3,0.4,0.4)\rangle$, then:

FNIFWG $_{\omega}\left(\tilde{a}_{1}, \tilde{a}_{2}, \tilde{a}_{3}, \tilde{a}_{4}\right)=$ $\left\langle\left(0.1^{0.2} \times 0.4^{0.1} \times 0.4^{0.3} \times 0.3^{0.4}\right.\right.$, $0.2^{0.2} \times 0.5^{0.1} \times 0.4^{0.3} \times 0.4^{0.4}$ $\left.0.3^{0.2} \times 0.6^{0.1} \times 0.5^{0.3} \times 0.5^{0.4}\right)$, $\left(1-(1-0.5)^{0.2} \times(1-0.3)^{0.1} \times(1-0.4)^{0.3} \times(1-0.3)^{0.4} \mathrm{~J}\right.$ $1-(1-0.6)^{0.2} \times(1-0.3)^{0.1} \times(1-0.4)^{0.3} \times(1-0.4)^{0.4} \mathrm{~J}$ $\left.\left.1-(1-0.7)^{0.2} \times(1-0.4)^{0.1} \times(1-0.5)^{0.3} \times(1-0.4)^{0.4}\right)\right\rangle=$ $\langle(0.270,0.356,0.460),(0.625,0.562,0.495)\rangle$.

Definition 15. Let $\tilde{a}\left(x_{i}\right)=\left\langle\left(a\left(x_{i}\right), b\left(x_{i}\right), c\left(x_{i}\right)\right),\left(l\left(x_{i}\right), m\left(x_{i}\right), p\left(x_{i}\right)\right)\right\rangle(i=1,2, \cdots, n)$ be a collection of FNIFVs, An fuzzy number intuitionistic fuzzy ordered weighted geometric (FNIFOWG) operator of dimension $n$ is a mapping FNIFOWG: $Q^{n} \rightarrow Q$, that has an associated weight vector $w=\left(w_{1}, w_{2}, \cdots, w_{n}\right)^{T}$ such that $w_{i}>0$ and $\sum_{i=1}^{n} w_{i}=1$.
Furthermore:

$$
\begin{aligned}
& \operatorname{FNIFOWG}_{w}\left(\tilde{a}\left(x_{1}\right), \tilde{a}\left(x_{2}\right), \cdots, \tilde{a}\left(x_{n}\right)\right)=\prod_{i=1}^{n} \tilde{a}\left(x_{\sigma(i)}\right)^{w_{i}}= \\
& \left\langle\left(\prod_{i=1}^{n} a\left(x_{\sigma(i)}\right)^{w_{i}}, \prod_{i=1}^{n} b\left(x_{\sigma(i)}\right)^{w_{i}}, \prod_{i=1}^{n} c\left(x_{\sigma(i)}\right)^{w_{i}}\right),\right. \\
& \left.\left(1-\prod_{i=1}^{n}\left(1-l\left(x_{\sigma(i)}\right)\right)^{w_{i}}, 1-\prod_{i=1}^{n}\left(1-m\left(x_{\sigma(i)}\right)\right)^{w_{i}}, 1-\prod_{i=1}^{n}\left(1-p\left(x_{\sigma(i)}\right)\right)^{w_{i}}\right)\right\rangle,
\end{aligned}
$$

where $(\sigma(1), \sigma(2), \cdots, \sigma(n))$ is a permutation of $(1,2, \cdots, n)$, such that $\tilde{a}\left(x_{\sigma(i-1)}\right) \geq \tilde{a}\left(x_{\sigma(i)}\right)$ for all $i=2, \cdots, n$.

Example 5. Let $\tilde{a}_{1}=\langle(0.3,0.4,0.5),(0.2,0.3,0.4)\rangle, \tilde{a}_{2}=\langle(0.3,0.3,0.3),(0.4,0.5,0.6)\rangle$, $\tilde{a}_{3}=\langle(0.5,0.5,0.5),(0.3,0.3,0.3)\rangle$, and $\tilde{a}_{4}=\langle(0.1,0.2,0.2),(0.6,0.7,0.8)\rangle$ be four FNIFVs, by (4), we calculate the scores of $\tilde{a}_{j}(j=1,2,3,4)$ :

$S\left(\tilde{a}_{1}\right)=0.1, S\left(\tilde{a}_{2}\right)=-0.2$,

$S\left(\tilde{a}_{3}\right)=0.2, S\left(\tilde{a}_{4}\right)=-0.53$.

Since

$S\left(\tilde{a}_{3}\right)>S\left(\tilde{a}_{1}\right)>S\left(\tilde{a}_{2}\right)>S\left(\tilde{a}_{4}\right)$,

thus 


$$
\begin{aligned}
& \tilde{a}_{\sigma(1)}=\langle(0.5,0.5,0.5),(0.3,0.3,0.3)\rangle, \tilde{a}_{\sigma(2)}=\langle(0.3,0.4,0.5),(0.2,0.3,0.4)\rangle, \\
& \tilde{a}_{\sigma(3)}=\langle(0.3,0.3,0.3),(0.4,0.5,0.6)\rangle, \tilde{a}_{\sigma(4)}=\langle(0.1,0.2,0.2),(0.6,0.7,0.8)\rangle .
\end{aligned}
$$

Suppose that $w=(0.2,0.3,0.4,0.1)$ is the weighting vector of the FIFOWG operator. Then, by (11), it follows that:

FNIFOWG $_{\omega}\left(\tilde{a}_{1}, \tilde{a}_{2}, \tilde{a}_{3}, \tilde{a}_{4}\right)=$

$\left\langle\left(0.5^{0.2} \times 0.3^{0.3} \times 0.3^{0.4} \times 0.1^{0.1}\right.\right.$,

$0.5^{0.2} \times 0.4^{0.3} \times 0.3^{0.4} \times 0.2^{0.1}$

$\left.0.5^{0.2} \times 0.5^{0.3} \times 0.3^{0.4} \times 0.2^{0.1}\right)$,

$\left(1-(1-0.3)^{0.2} \times(1-0.2)^{0.3} \times(1-0.4)^{0.4} \times(1-0.6)^{0.1} \mathrm{~J}\right.$

$1-(1-0.3)^{0.2} \times(1-0.3)^{0.3} \times(1-0.5)^{0.4} \times(1-0.7)^{0.1} \mathrm{~J}$

$\left.\left.1-(1-0.3)^{0.2} \times(1-0.4)^{0.3} \times(1-0.6)^{0.4} \times(1-0.8)^{0.1}\right)\right\rangle=$

$\langle(0.214,0.291,0.297),(0.541,0.451,0.353)\rangle$.

In the following, we shall develop the induced fuzzy number intuitionistic fuzzy choquet ordered geometric (IFNIFCOG) operator based on the well-known Choquet integral (Choquet 1953).

Definition 16. Let $X\left(x_{1}, x_{2}, \cdots, x_{n}\right)$ be a finite set, and $\mu$ be a fuzzy measure on $X$, and $\tilde{a}\left(x_{i}\right)=\left\langle\left(a\left(x_{i}\right), b\left(x_{i}\right), c\left(x_{i}\right)\right),\left(l\left(x_{i}\right), m\left(x_{i}\right), p\left(x_{i}\right)\right)\right\rangle \quad(i=1,2, \cdots, n)$ be a collection of FNIFVs on $X$, and $\mu$ be a fuzzy measure on $X$. An induced fuzzy number intuitionistic fuzzy choquet ordered geometric (IFNIFCOG) operator of dimension $n$ is a function IFNIFCOG: $Q^{n} \rightarrow Q$, which is defined to aggregate the set of second arguments of a collection of 2-tuples $\left(\left\langle u_{1}, \tilde{a}\left(x_{1}\right)\right\rangle,\left\langle u_{2}, \tilde{a}\left(x_{2}\right)\right\rangle, \cdots,\left\langle u_{n}, \tilde{a}\left(x_{n}\right)\right\rangle\right)$ according to the following expression:

$$
\begin{aligned}
& \operatorname{IFNIFCOG}_{\mu}\left(\left\langle u_{1}, \tilde{a}\left(x_{1}\right)\right\rangle,\left\langle u_{2}, \tilde{a}\left(x_{2}\right)\right\rangle, \cdots,\left\langle u_{n}, \tilde{a}\left(x_{n}\right)\right\rangle\right)= \\
& \tilde{a}\left(x_{\sigma(1)}\right)^{\mu\left(A_{\sigma(1)}\right)-\mu\left(A_{\sigma(0)}\right)} \otimes a\left(\tilde{x}_{\sigma(2)}\right)^{\mu\left(A_{\sigma(2)}\right)-\mu\left(A_{\sigma(1)}\right)} \otimes \cdots \otimes a\left(\tilde{x}_{\sigma(n)}\right)^{\mu\left(A_{\sigma(n)}\right)-\mu\left(A_{\sigma(n-1)}\right)}= \\
& \bigotimes_{j=1}^{n} a\left(\tilde{x}_{\sigma(j)}\right)^{\mu\left(A_{\sigma(j)}\right)-\mu\left(A_{\sigma(j-1)}\right)},
\end{aligned}
$$

where $u_{i}$ in 2-tuple $\left\langle u_{i}, \tilde{a}\left(x_{i}\right)\right\rangle$ is referred to as the order-inducing variable and $\tilde{a}\left(x_{i}\right)$ as the argument variable, $(\sigma(1), \sigma(2), \cdots, \sigma(n))$ is a permutation of $(1,2, \cdots, n)$, such that $u_{\sigma(j-1)} \geq u_{\sigma(j)}$ for all $j=2, \cdots, n, A_{(i)}=\left\{x_{(1)}, x_{(2)}, \cdots, x_{(i)}\right\}$ when $i \geq 1$ and $A_{\sigma(0)}=\phi$. 
With the operation of fuzzy number intuitionistic fuzzy numbers, the IFNIFCOG operator can be transformed into the following from by induction on $n$ :

$$
\begin{aligned}
& \operatorname{IFNIFCOG}_{\mu}\left(\left\langle u_{1}, \tilde{a}\left(x_{1}\right)\right\rangle,\left\langle u_{2}, \tilde{a}\left(x_{2}\right)\right\rangle, \cdots,\left\langle u_{n}, \tilde{a}\left(x_{n}\right)\right\rangle\right)= \\
& \tilde{a}\left(x_{\sigma(1)}\right)^{\mu\left(A_{\sigma(1)}\right)-\mu\left(A_{\sigma(0)}\right)} \otimes a\left(\tilde{x}_{\sigma(2)}\right)^{\mu\left(A_{\sigma(2)}\right)-\mu\left(A_{\sigma(1)}\right)} \otimes \cdots \otimes a\left(\tilde{x}_{\sigma(n)}\right)^{\mu\left(A_{\sigma(n)}\right)-\mu\left(A_{\sigma(n-1)}\right)}= \\
& \bigotimes_{j=1}^{n} a\left(\tilde{x}_{\sigma(j)}\right)^{\mu\left(A_{\sigma(j)}\right)-\mu\left(A_{\sigma(j-1)}\right)}= \\
& \left\langle\left(\prod_{j=1}^{n}\left(a\left(x_{\sigma(i)}\right)\right)^{\mu\left(A_{\sigma(i)}\right)-\mu\left(A_{\sigma(i-1)}\right)}, \prod_{j=1}^{n}\left(b\left(x_{\sigma(i)}\right)\right)^{\mu\left(A_{\sigma(i)}\right)-\mu\left(A_{\sigma(i-1)}\right)}, \prod_{j=1}^{n}\left(c\left(x_{\sigma(i)}\right)\right)^{\mu\left(A_{\sigma(i)}\right)-\mu\left(A_{\sigma(i-1)}\right)}\right),\right. \\
& \left.\left(1-\prod_{i=1}^{n}\left(1-l\left(x_{\sigma(i)}\right)\right)^{\mu\left(A_{\sigma(i)}\right)-\mu\left(A_{\sigma(i-1)}\right)}, 1-\prod_{i=1}^{n}\left(1-m\left(x_{\sigma(i)}\right)\right)^{\mu\left(A_{\sigma(i)}\right)-\mu\left(A_{\sigma(i-1)}\right)}, 1-\prod_{i=1}^{n}\left(1-p\left(x_{\sigma(i)}\right)\right)^{\mu\left(A_{\sigma(i)}\right)-\mu\left(A_{\sigma(i-1)}\right)}\right)\right\rangle,
\end{aligned}
$$

whose aggregated value is also a fuzzy number intuitionistic fuzzy number.

Especially, if $\mu\left(\left\{x_{\sigma(j)}\right\}\right)=\mu\left(A_{\sigma(j)}\right)-\mu\left(A_{\sigma(j-1)}\right), i=1,2, \cdots, n$, then IFNIFCOG operator reduce to IFNIFWG operator. If $\mu(A)=\sum_{x_{j} \in A} \mu\left(\left\{x_{j}\right\}\right)$, for all $A \subseteq X$, where $|A|$ is the number of the elements in the set $A$, then $x_{j}=\mu\left(A_{\sigma(j)}\right)-\mu\left(A_{\sigma(j-1)}\right), i=1,2, \cdots, n$, where $w=\left(w_{1}, w_{2}, \cdots, w_{n}\right)^{T}, w_{j} \geq 0, i=1,2, \cdots, n$, and $\sum_{j=1}^{n} w_{j}=1$, then, IFNIFCOG op-
erator reduce to FNIFOWG operator. It's easy to prove that the IFNIFCOG operator has the following properties.

Theorem 4 (Commutativity).

$\operatorname{IFNIFCOG}_{\mu}\left(\tilde{a}\left(x_{1}\right), \tilde{a}\left(x_{2}\right), \cdots, \tilde{a}\left(x_{n}\right)\right)=$

$\operatorname{IFNIFCOG}_{\mu}\left(\tilde{a}^{\prime}\left(x_{1}\right), \tilde{a}^{\prime}\left(x_{2}\right), \cdots, \tilde{a}^{\prime}\left(x_{n}\right)\right)$, where $\left(\tilde{a}^{\prime}\left(x_{1}\right), \tilde{a}^{\prime}\left(x_{2}\right), \cdots, \tilde{a}^{\prime}\left(x_{n}\right)\right)$ is any permutation of $\left(\tilde{a}\left(x_{1}\right), \tilde{a}\left(x_{2}\right), \cdots, \tilde{a}\left(x_{n}\right)\right)$.

Theorem 5 (Idempotency). If $\tilde{a}\left(x_{j}\right)=\tilde{a}(x)$ for all $j$, then:

$\operatorname{IFNIFCOG}_{\mu}\left(\tilde{a}\left(x_{1}\right), \tilde{a}\left(x_{2}\right), \cdots, \tilde{a}\left(x_{n}\right)\right)=\tilde{a}(x)$.

Theorem 6 (Monotonicity). If $\tilde{a}\left(x_{j}\right) \leq \tilde{a}^{\prime}\left(x_{j}\right)$ for all $j$, then:

$\operatorname{IFNIFCOG}_{\mu}\left(\tilde{a}\left(x_{1}\right), \tilde{a}\left(x_{2}\right), \cdots, \tilde{a}\left(x_{n}\right)\right) \leq$

$\operatorname{IFNIFCOG}_{\mu}\left(\tilde{a}^{\prime}\left(x_{1}\right), \tilde{a}^{\prime}\left(x_{2}\right), \cdots, \tilde{a}^{\prime}\left(x_{n}\right)\right)$.

Example 6. Assume we have four FNIFOWG pairs $\left\langle u_{j}, \tilde{a}\left(x_{j}\right)\right\rangle$ given:

$\left\langle u_{1}, \tilde{a}\left(x_{1}\right)\right\rangle=\langle 12,((0.3,0.4,0.5),(0.1,0.2,0.3))\rangle$,

$\left\langle u_{2}, \tilde{a}\left(x_{2}\right)\right\rangle=\langle 15,((0.4,0.5,0.5),(0.1,0.1,0.2))\rangle$, 


$$
\begin{aligned}
& \left\langle u_{3}, \tilde{a}\left(x_{3}\right)\right\rangle=\langle 10,((0.2,0.3,0.3),(0.3,0.4,0.5))\rangle, \\
& \left\langle u_{4}, \tilde{a}\left(x_{4}\right)\right\rangle=\langle 8,((0.2,0.3,0.4),(0.4,0.5,0.6))\rangle,
\end{aligned}
$$

that we desire to aggregate using the weighting vector $w=(0.2,0.4,0.1,0.3)$. Performing the ordering the FNIFOWG pairs with respect to the first compoent, we get:

$$
\begin{aligned}
&\left\langle u_{\sigma(1)}, \tilde{a}\left(x_{\sigma(1)}\right)\right\rangle=\langle 15,((0.4,0.5,0.5),(0.1,0.1,0.2))\rangle, \\
&\left.u_{\sigma(2)}, \tilde{a}\left(x_{\sigma(2)}\right)\right\rangle=\langle 12,((0.3,0.4,0.5),(0.1,0.2,0.3))\rangle, \\
&\left.u_{\sigma(3)}, \tilde{a}\left(x_{\sigma(3)}\right)\right\rangle=\langle 10,((0.2,0.3,0.3),(0.3,0.4,0.5))\rangle, \\
&\left.u_{\sigma(4)}, \tilde{a}\left(x_{\sigma(4)}\right)\right\rangle=\langle 8,((0.2,0.3,0.4),(0.4,0.5,0.6))\rangle .
\end{aligned}
$$

This ordering includes the ordered fuzzy number intuitionistic fuzzy numbers:

$$
\begin{aligned}
& \tilde{a}\left(x_{\sigma(1)}\right)=\langle(0.4,0.5,0.5),(0.1,0.1,0.2)\rangle, \\
& \tilde{a}\left(x_{\sigma(2)}\right)=\langle(0.3,0.4,0.5),(0.1,0.2,0.3)\rangle, \\
& \tilde{a}\left(x_{\sigma(3)}\right)=\langle(0.2,0.3,0.3),(0.3,0.4,0.5)\rangle, \\
& \tilde{a}\left(x_{\sigma(4)}\right)=\langle(0.2,0.3,0.4),(0.4,0.5,0.6)\rangle .
\end{aligned}
$$

Suppose the fuzzy measure of attribute of $\tilde{a}_{i}(i=1,2,3,4)$ and attribute sets of $\tilde{a}_{i}(i=1,2,3,4)$ as follows:

$$
\begin{aligned}
& \mu(\phi)=0, \mu\left(\tilde{a}_{1}\right)=0.40, \mu\left(\tilde{a}_{2}\right)=0.25, \mu\left(\tilde{a}_{3}\right)=0.38, \mu\left(\tilde{a}_{4}\right)=0.25, \\
& \mu\left(\tilde{a}_{1}, \tilde{a}_{2}\right)=0.56, \mu\left(\tilde{a}_{1}, \tilde{a}_{3}\right)=0.65, \mu\left(\tilde{a}_{1}, \tilde{a}_{4}\right)=0.50, \mu\left(\tilde{a}_{2}, \tilde{a}_{3}\right)=0.45, \\
& \mu\left(\tilde{a}_{2}, \tilde{a}_{4}\right)=0.39, \mu\left(\tilde{a}_{3}, \tilde{a}_{4}\right)=0.40, \mu\left(\tilde{a}_{1}, \tilde{a}_{2}, \tilde{a}_{3}\right)=0.80, \mu\left(\tilde{a}_{1}, \tilde{a}_{2}, \tilde{a}_{4}\right)=0.75, \\
& \mu\left(\tilde{a}_{1}, \tilde{a}_{3}, \tilde{a}_{4}\right)=0.72, \mu\left(\tilde{a}_{2}, \tilde{a}_{3}, \tilde{a}_{4}\right)=0.60, \mu\left(\tilde{a}_{1}, \tilde{a}_{2}, \tilde{a}_{3}, \tilde{a}_{4}\right)=1.00 .
\end{aligned}
$$

Then, by (14), it follows that:

$$
\begin{aligned}
& \operatorname{IFNIFCOG}_{\mu}\left(\left\langle u_{1}, \tilde{a}\left(x_{1}\right)\right\rangle,\left\langle u_{2}, \tilde{a}\left(x_{2}\right)\right\rangle,\left\langle u_{3}, \tilde{a}\left(x_{3}\right)\right\rangle,\left\langle u_{4}, \tilde{a}\left(x_{4}\right)\right\rangle\right)= \\
& \left\langle\left( 0.4^{0.25} \times 0.3^{0.56-0.25} \times 0.2^{0.80-0.56} \times 0.1^{1-0.80},\right.\right. \\
& 0.5^{0.25} \times 0.4^{0.56-0.25} \times 0.3^{0.80-0.56} \times 0.2^{1-0.80}, \\
& \left.0.5^{0.25} \times 0.5^{0.56-0.25} \times 0.3^{0.80-0.56} \times 0.3^{1-0.80}\right), \\
& \left(1-(1-0.1)^{0.25} \times(1-0.1)^{0.56-0.25} \times(1-0.3)^{0.80-0.56} \times(1-0.3)^{1-0.80} \mathrm{~J}\right. \\
& 1-(1-0.1)^{0.25} \times(1-0.2)^{0.56-0.25} \times(1-0.4)^{0.80-0.56} \times(1-0.4)^{1-0.80} \mathrm{~J} \\
& \left.\left.1-(1-0.2)^{0.25} \times(1-0.3)^{0.56-0.25} \times(1-0.5)^{0.80-0.56} \times(1-0.5)^{1-0.80}\right)\right\rangle= \\
& \langle(0.292,0.344,0.399),(0.194,0.274,0.661)\rangle .
\end{aligned}
$$




\section{An approach to multiple attribute decision making with fuzzy number intuitionistic fuzzy information}

In this section, we shall develop an approach to multiple attribute decision making with fuzzy number intuitionistic fuzzy information as follows.

Let $A=\left\{A_{1}, A_{2}, \cdots, A_{m}\right\}$ be a discrete set of alternatives, and $G=\left\{G_{1}, G_{2}, \cdots, G_{n}\right\}$ be the set of attributes, $\omega=\left(\omega_{1}, \omega_{2}, \cdots, \omega_{n}\right)$ is the weighting vector of the attribute $G_{j}(j=1,2, \cdots, n)$, where $\omega_{j} \in[0,1], \sum_{j=1}^{n} \omega_{j}=1$.Suppose that $\tilde{R}=\left(\tilde{r}_{i j}\right)_{m \times n}=\left\langle\left(a_{i j}, b_{i j}, c_{i j}\right),\left(l_{i j}, m_{i j}, p_{i j}\right)\right\rangle_{m \times n}$ is the fuzzy number intuitionistic fuzzy decision matrix, where $\left(a_{i j}, b_{i j}, c_{i j}\right)$ indicates the degree that the alternative $A_{i}$ satisfies the attribute $G_{j},\left(l_{i j}, m_{i j}, p_{i j}\right)$ indicates the degree that the alternative $A_{i}$ doesn't satisfy the attribute $G_{j}\left(a_{i j}, b_{i j}, c_{i j}\right) \subset[0,1]$, $\left(l_{i j}, m_{i j}, p_{i j}\right) \subset[0,1], c_{i j}+p_{i j} \leq 1, i=1,2, \cdots, m, j=1,2, \cdots, n$.

In the following, we apply the IFNIFCOA and IFNIFCOG operator to multiple attribute decision making with fuzzy number intuitionistic fuzzy information.

Step 1. Calculate the order-inducing variables $\left(u_{i j}\right)_{m \times n}$ to be used in the decision matrix for each alternative $A_{i}$ and the attribute $G_{j}$. The experts use order-inducing variables to represent the complex attitudinal character involving the opinion of different members of the board of directors (Merigó, Gil-Lafuente 2009).

Step 2. If we emphasize the group's influence, utilize the decision information given in matrix $\tilde{R}$, and the IFNIFCOA operator:

$$
\begin{aligned}
& \tilde{r}_{i}=\left\langle\left(a_{j}, b_{j}, c_{j}\right),\left(l_{j}, m_{j}, p_{j}\right)\right\rangle= \\
& \operatorname{IFNIFCOA}\left(\left\langle u_{i 1}, \tilde{a}\left(x_{i 1}\right)\right\rangle,\left\langle u_{i 2}, \tilde{a}\left(x_{i 2}\right)\right\rangle, \cdots,\left\langle u_{i n}, \tilde{a}\left(x_{i n}\right)\right\rangle\right), \\
& i=1,2, \cdots, m, j=1,2, \cdots, n
\end{aligned}
$$

to derive the overall preference values $\tilde{r}_{i}(i=1,2, \cdots, m)$ of the alternative $A_{i}$. Otherwise utilize the decision information given in matrix $\tilde{R}$, and the IFNIFCOG operator:

$$
\begin{aligned}
& \tilde{r}_{i}=\left\langle\left(a_{j}, b_{j}, c_{j}\right),\left(l_{j}, m_{j}, p_{j}\right)\right\rangle= \\
& \operatorname{IFNIFCOG}\left(\left\langle u_{i 1}, \tilde{a}\left(x_{i 1}\right)\right\rangle,\left\langle u_{i 2}, \tilde{a}\left(x_{i 2}\right)\right\rangle, \cdots,\left\langle u_{i n}, \tilde{a}\left(x_{i n}\right)\right\rangle\right), \\
& i=1,2, \cdots, m, j=1,2, \cdots, n
\end{aligned}
$$

to derive the overall preference values $\tilde{r}_{i}(i=1,2, \cdots, m)$ of the alternative $A_{i}$.

Step 3. Calculate the scores $S\left(\tilde{r}_{i}\right)(i=1,2, \cdots, m)$ of the overall fuzzy number intuitionistic fuzzy preference values $\tilde{r}_{i}(i=1,2, \cdots, m)$ to rank all the alternatives $A_{i}(i=1,2, \cdots, m)$ and then to select the best one(s) (if there is no difference between two scores $S\left(\tilde{r}_{i}\right)$ and $S\left(\tilde{r}_{j}\right)$, then we need to calculate the accuracy degrees $H\left(\tilde{r}_{i}\right)$ and $H\left(\tilde{r}_{j}\right)$ of the overall fuzzy number intuitionistic fuzzy preference values $\tilde{r}_{i}$ and $\tilde{r}_{j}$, respectively, and then rank the alternatives $A_{i}$ and $A_{j}$ in accordance with the accuracy degrees $H\left(\tilde{r}_{i}\right)$ and $H\left(\tilde{r}_{j}\right)$. 
Step 4. Rank all the alternatives $A_{i}(i=1,2, \cdots, m)$ and select the best one(s) in accordance with $S\left(\tilde{r}_{i}\right)$ and $H\left(\tilde{r}_{i}\right)(i=1,2, \cdots, m)$.

Step 5. End.

\section{Illustrative example}

In this section we shall present a numerical example to show potential evaluation of emerging technology commercialization with fuzzy number intuitionistic fuzzy information in order to illustrate the method proposed in this paper. There is a panel with five possible emerging technology enterprises $A_{i}(i=1,2,3,4,5)$ to select. The experts selects four attribute to evaluate the four possible emerging technology enterprises: (1) $\mathrm{G}_{1}$ is the technical advancement; (2) $\mathrm{G}_{2}$ is the potential market and market risk; (3) $\mathrm{G}_{3}$ is the industrialization infrastructure, human resources and financial conditions; (4) $\mathrm{G}_{4}$ is the employment creation and the development of science and technology. The five possible enterprises $A_{i}(i=1,2,3,4,5)$ are to be evaluated using using the fuzzy number intuitionistic fuzzy numbers by the decision maker under the above four attributes, and construct, respectively, the decision matrices as listed in the following matrices $\tilde{R}_{k}=\left(\tilde{r}_{i j}^{(k)}\right)_{5 \times 4}(k=1,2,3)$ as follows:

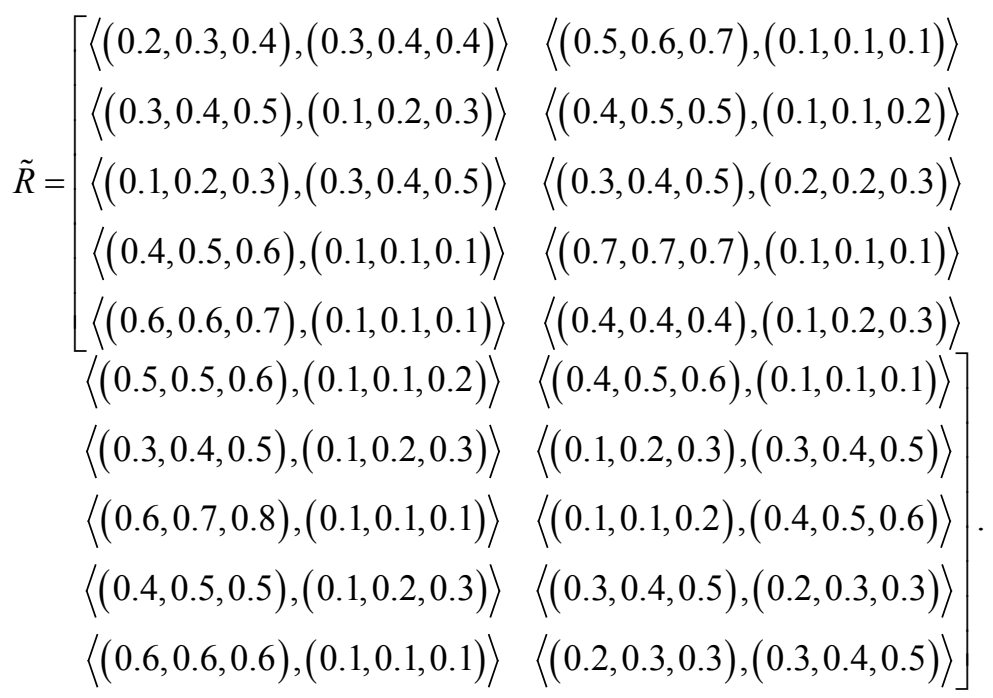

Then, we utilize the approach developed to get the most desirable alternative(s).

Step 1. Suppose the fuzzy measure of attribute of $G_{j}(j=1,2, \cdots, n)$ and attribute sets of $G$ as follows:

$$
\begin{aligned}
& \mu\left(G_{1}\right)=0.30, \mu\left(G_{2}\right)=0.35, \mu\left(G_{3}\right)=0.30, \mu\left(G_{4}\right)=0.22, \mu\left(G_{1}, G_{2}\right)=0.70, \\
& \mu\left(G_{1}, G_{3}\right)=0.60, \mu\left(G_{1}, G_{4}\right)=0.55, \mu\left(G_{2}, G_{3}\right)=0.50, \mu\left(G_{2}, G_{4}\right)=0.45, \\
& \mu\left(G_{3}, G_{4}\right)=0.40, \mu\left(G_{1}, G_{2}, G_{3}\right)=0.82, \mu\left(G_{1}, G_{2}, G_{4}\right)=0.87, \\
& \mu\left(G_{1}, G_{3}, G_{4}\right)=0.75, \mu\left(G_{2}, G_{3}, G_{4}\right)=0.60, \mu\left(G_{1}, G_{2}, G_{3}, G_{4}\right)=1.00 .
\end{aligned}
$$


Step 2. The experts use order-inducing variables to represent the complex attitudinal character involving the opinion of different members of the board of directors (Merigó, Gil-Lafuente 2009). The results are shown in Table 1.

Table 1. Inducing variables

\begin{tabular}{lllll}
\hline & $S_{1}$ & $S_{2}$ & $S_{3}$ & $S_{4}$ \\
\hline$A_{1}$ & 12 & 18 & 16 & 15 \\
\hline$A_{2}$ & 23 & 25 & 22 & 20 \\
\hline$A_{3}$ & 18 & 20 & 24 & 14 \\
\hline$A_{4}$ & 22 & 24 & 20 & 18 \\
\hline$A_{5}$ & 24 & 18 & 21 & 16 \\
\hline
\end{tabular}

Step 3. If we emphasize the group's influence, we utilize the decision information given in matrix $\tilde{R}$, and the IFNIFCOA operator to obtain the overall preference values $\tilde{r}_{i}$ of the alternatives $A_{i}(i=1,2, \cdots, 5)$.

$$
\begin{aligned}
& \tilde{r}_{1}=\langle(0.385,0.471,0.575),(0.155,0.174,0.193)\rangle, \\
& \tilde{r}_{2}=\langle(0.306,0.407,0.469),(0.122,0.178,0.285)\rangle, \\
& \tilde{r}_{3}=\langle(0.329,0.425,0.540),(0.210,0.239,0.288)\rangle, \\
& \tilde{r}_{4}=\langle(0.516,0.568,0.613),(0.113,0.132,0.139)\rangle, \\
& \tilde{r}_{5}=\langle(0.505,0.516,0.556),(0.122,0.149,0.170)\rangle .
\end{aligned}
$$

Step 4. Calculate the scores $S\left(\tilde{r}_{i}\right)(i=1,2, \cdots, 5)$ of the overall fuzzy number intuitionistic fuzzy preference values $\tilde{r}_{i}(i=1,2, \cdots, 5)$ :

$S\left(\tilde{r}_{1}\right)=0.603, S\left(\tilde{r}_{2}\right)=0.413, S\left(\tilde{r}_{3}\right)=0.372, S\left(\tilde{r}_{4}\right)=0.874, S\left(\tilde{r}_{5}\right)=0.751$.

Step 5. Rank all the alternatives $A_{i}(i=1,2,3,4,5)$ in accordance with the scores $S\left(\tilde{r}_{i}\right)(i=1,2, \cdots, 5)$ of the overall fuzzy number intuitionistic fuzzy preference values $\tilde{r}_{i}(i=1,2, \cdots, 5): A_{4} \succ A_{5} \succ A_{1} \succ A_{2} \succ A_{3}$, and thus the most desirable alternative is $A_{4}$. If we emphasize the individual influence, we utilize the decision information given in matrix $\tilde{R}$ and the IFNIFCOG operator to obtain the overall preference values $\tilde{r}_{i}$ of the alternatives $A_{i}(i=1,2, \cdots, 5)$.

$$
\begin{aligned}
& \tilde{r}_{1}=\langle(0.339,0.434,0.538),(0.186,0.235,0.248)\rangle, \\
& \tilde{r}_{2}=\langle(0.272,0.382,0.456),(0.140,0.208,0.310)\rangle, \\
& \tilde{r}_{3}=\langle(0.213,0.295,0.415),(0.246,0.305,0.387)\rangle, \\
& \tilde{r}_{4}=\langle(0.462,0.540,0.600),(0.119,0.152,0.165)\rangle, \\
& \tilde{r}_{5}=\langle(0.450,0.484,0.507),(0.140,0.185,0.234)\rangle .
\end{aligned}
$$


Then, by applying Eqs (4) to calculate the scores $S\left(\tilde{r}_{i}\right)(i=1,2, \cdots, 5)$ of the collective overall fuzzy number intuitionistic fuzzy preference values $\tilde{r}_{i}(i=1,2, \cdots, 5)$ :

$S\left(\tilde{r}_{1}\right)=0.421, S\left(\tilde{r}_{2}\right)=0.313, S\left(\tilde{r}_{3}\right)=-0.013, S\left(\tilde{r}_{4}\right)=0.777, S\left(\tilde{r}_{5}\right)=0.592$.

Therefore, the ranking order is $A_{4} \succ A_{5} \succ A_{1} \succ A_{2} \succ A_{3}$. Thus, we can see that the most desirable alternative is still $A_{4}$.

Especially, if the triangular fuzzy numbers $\left(a_{j}, b_{j}, c_{j}\right)$ and $\left(l_{j}, m_{j}, p_{j}\right)$ are reduced to the interval numbers $\left[a_{j}, b_{j}\right]$ and $\left[l_{j}, m_{j}\right]$, then, the IFNIFCOA or IFNIFCOG operator is reduced to the induced interval-valued intuitionistic fuzzy choquet ordered averaging (I-IVIFCOA) operator or induced interval-valued intuitionistic fuzzy choquet ordered geometric (I-IVIFCOG) operator (Xu, Xia 2011); if $a_{j}=b_{j}=c_{j}=\mu_{j}$, $l_{j}=m_{j}=p_{j}=v_{j}$, then the IFNIFCOA or IFNIFCOG operator is reduced to the induced intuitionistic fuzzy choquet ordered averaging (I-IFCOA) operator or induced intuitionistic fuzzy choquet ordered geometric (I-IFCOG) operator (Xu, Xia 2011).

\section{Conclusions}

The traditional induced Choquet integral aggregation operators are generally suitable for aggregating the information taking the form of numerical values, and yet they will fail in dealing with fuzzy number intuitionistic fuzzy information. In this paper, we have developed two induced fuzzy number intuitionistic fuzzy Choquet integral aggregation operators: induced fuzzy number intuitionistic fuzzy choquet ordered averaging (IFNIFCOA) operator and induced fuzzy number intuitionistic fuzzy choquet ordered geometric (IFNIFCOG) operator. The prominent characteristic of the operators is that they can not only consider the importance of the elements or their ordered positions, but also reflect the correlation among the elements or their ordered positions. We have studied some desirable properties of the IFNIFCOA and IFNIFCOG operators, such as commutativity, idempotency and monotonicity, and applied the IFNIFCOA and IFNIFCOGM operators to multiple attribute decision making with fuzzy number intuitionistic fuzzy information. Finally an illustrative example has been given to show the developed method. In the future, we shall continue working in the application of the fuzzy number intuitionistic fuzzy multiple attribute decision making to other domains.

\section{Acknowledgment}

The author is very grateful to the editor and the anonymous referees for their insightful and constructive comments and suggestions, which have been very helpful in improving the paper. The work was supported by the National Natural Science Foundation of China under Grant No. 61174149, Natural Science Foundation Project of CQ CSTC of the People's Republic of China (No. CSTC, 2011BA0035) and the Humanities and Social Sciences Foundation of Ministry of Education of the People's Republic of China under Grant No.11XJC630011. 


\section{References}

Atanassov, K. 1986. Intuitionistic fuzzy sets, Fuzzy Sets and Systems 20: 87-96. http://dx.doi. org/10.1016/S0165-0114(86)80034-3

Atanassov, K.; Gargov, G. 1989. Interval valued intuitionistic fuzzy sets, Fuzzy Sets and Systems 31: 343-349. http://dx.doi.org/10.1016/0165-0114(89)90205-4

Atanassov, K. 1994. Operators over interval-valued intuitionistic fuzzy sets, Fuzzy Sets and Systems 64: 159-174. http://dx.doi.org/10.1016/0165-0114(94)90331-X

Choquet, G. 1953. Theory of capacities, Annales de l'Institut Fourier 5: 131-295.

http://dx.doi.org/10.5802/aif.53

Geng, X. L.; Chu, X. N.; Zhang, Z. F. 2010. A new integrated design concept evaluation approach based on vague sets, Expert Systems with Applications 37(9): 6629-6638.

http://dx.doi.org/10.1016/j.eswa.2010.03.058

Grabisch, M.; Murofushi, T.; Sugeno, M. 2000. Fuzzy measure and integrals. New York: PhysicaVerlag.

Kersuliene, V.; Zavadskas, E. K.; Turskis, Z. 2010. Selection of rational dispute resolution method by applying new stepwise weight assessment ratio analysis (SWARA), Journal of Business Economics and Management 11(2): 243-258. http://dx.doi.org/10.3846/jbem.2010.12

Keeney, R. L.; Raiffa, H. 1976. Decision with multiple objectives. New York: Wiley.

Li, D. F. 2010. Linear programming method for MADM with interval-valued intuitionistic fuzzy sets, Expert Systems with Applications 37(8): 5939-5945.

http://dx.doi.org/10.1016/j.eswa.2010.02.011

Liu, P.D. 2009. Multi-attribute decision-making method research based on interval vague set and TOPSIS method, Technological and Economic Development of Economy 15(3): 453-463.

http://dx.doi.org/10.3846/1392-8619.2009.15.453-463

Liu, F.; Yuan, X. H. 2007. Fuzzy number intuitionistic fuzzy set, Fuzzy Systems and Mathematics 21(1): 88-91.

Merigó, J. M.; Gil-Lafuente, A. M. 2009. The induced generalized OWA operator, Information Sciences 179(6): 729-741. http://dx.doi.org/10.1016/j.ins.2008.11.013

Wachowicz, T. 2010. Decision support in software supported negotiations, Journal of Business Economics and Management 11(4): 576-597. http://dx.doi.org/10.3846/jbem.2010.28

Wakker, P. 1999. Additive representations of preferences. Kluwer Academic Publishers.

Wang, X. F. 2008a. Fuzzy number intuitionistic fuzzy arithmetic aggregation operators, International Journal of Fuzzy Systems 10(2): 104-111.

Wang, X. F. 2008b. Fuzzy number intuitionistic fuzzy geometric aggregation operators and their application to decision making, Control and decision 23(6): 607-612.

Wang, Z.; Klir, G. 1992. Fuzzy measure theory. New York: Plenum Press.

http://dx.doi.org/10.1007/978-1-4757-5303-5

Wei, G. W. 2008. Maximizing deviation method for multiple attribute decision making in intuitionistic fuzzy setting, Knowledge-Based Systems 21(8): 833-836.

http://dx.doi.org/10.1016/j.knosys.2008.03.038

Wei, G. W. 2009. Some geometric aggregation functions and their application to dynamic multiple attribute decision making in intuitionistic fuzzy setting, International Journal of Uncertainty, Fuzziness and Knowledge-Based Systems 17(2): 179-196.

http://dx.doi.org/10.1142/S0218488509005802

Wei, G. W. 2010a. Some induced geometric aggregation operators with intuitionistic fuzzy information and their application to group decision making, Applied Soft Computing 10(2): 423-431. http://dx.doi.org/10.1016/j.asoc.2009.08.009 
Wei, G. W. 2010b. GRA method for multiple attribute decision making with incomplete weight information in intuitionistic fuzzy setting, Knowledge-Based Systems 23(3): 243-247.

http://dx.doi.org/10.1016/j.knosys.2010.01.003

Wei, G. W.; Zhao, X. F.; Lin, R. 2010a. Some induced aggregating operators with fuzzy number intuitionistic fuzzy information and their applications to group decision making, International Journal of Computational Intelligence Systems 3(1): 84-95.

Wei, G. W.; Wang, H. J.; Lin, R. 2010b. Application of correlation coefficient to interval-valued intuitionistic fuzzy multiple attribute decision making with incomplete weight information, Knowledge and Information Systems 26(2): 337-349.

http://dx.doi.org/10.1007/s10115-009-0276-1

Xu, Z. S. 2007a. Intuitionistic fuzzy aggregation operators, IEEE Transactions on Fuzzy Systems 15: 1179-1187. http://dx.doi.org/10.1109/TFUZZ.2006.890678

$\mathrm{Xu}, \mathrm{Z}$. S. 2007b. Methods for aggregating interval-valued intuitionistic fuzzy information and their application to decision making, Control and Decision 22(2): 215-219.

$\mathrm{Xu}, \mathrm{Z}$. S.; Chen, J. 2007. An approach to group decision making based on interval-valued intuitionistic fuzzy judgment matrices, System Engineer-Theory and Practice 27(4): 126-133.

http://dx.doi.org/10.1016/S1874-8651(08)60026-5

Xu, Z. S.; Yager, R. R. 2006. Some geometric aggregation operators based on intuitionistic fuzzy sets, International Journal of General System 35: 417-433.

http://dx.doi.org/10.1080/03081070600574353

Xu, Z. S.; Xia, M. M. 2011. Induced generalized intuitionistic fuzzy operators, Knowledge-Based Systems 24: 197-209. http://dx.doi.org/10.1016/j.knosys.2010.04.010

Ye, J. 2009. Multicriteria fuzzy decision-making method based on a novel accuracy function under interval-valued intuitionistic fuzzy environment, Expert Systems with Applications 36(3): 6899-6902. http://dx.doi.org/10.1016/j.eswa.2008.08.042

Zadeh, L. A. 1965. Fuzzy sets, Information and Control 8: 338-353.

http://dx.doi.org/10.1016/S0019-9958(65)90241-X

Zadeh, L. A. 2008. Is there a need for fuzzy logic?, Information Sciences 178(2008): 2751-2779. http://dx.doi.org/10.1016/j.ins.2008.02.012

Zavadskas, E. K.; Vilutiene, T.; Turskis, Z.; Tamosaitiene, J. 2010. Contractor selection for construction works by applying SAW-G and TOPSIS Grey techniques, Journal of Business Economics and Management 11(1): 34-55. http://dx.doi.org/10.3846/jbem.2010.03 
Guiwu WEI has a MSc and a PhD degree in applied mathematics from SouthWest Petroleum University, Business Administration from School of Economics and Management at SouthWest Jiaotong University, China, respectively. He is an Associate Professor in the Department of Economics and Management at Chongqing University of Arts and Sciences. He has published more than 90 papers in journals, books and conference proceedings including journals such as Expert Systems with Applications, Applied Soft Computing, Knowledge and Information Systems, Knowledge-based Systems, International Journal of Uncertainty, Fuzziness and Knowledge-Based Systems, International Journal of Computational Intelligence Systems and Information: An International Journal. He has published 1 book. He has participated in several scientific committees and serves as a reviewer in a wide range of journals including Computers \& Industrial Engineering, International Journal of Information Technology and Decision Making, Knowledge-based Systems, Information Sciences, International Journal of Computational Intelligence Systems and European Journal of Operational Research. He is currently interested in Aggregation Operators, Decision Making and Computing with Words.

Rui LIN is a lecturer in Department of Economics and Management, Chongqing University of Arts and Sciences. He received the B.E. degree in management sciences and engineer from Chengdu University of Technology, China. He has worked for Department of Economics and Management, Chongqing University of Arts and Sciences, China as a lecturer since 2007.

Xiaofei ZHAO is a lecturer in Department of Economics and Management, Chongqing University of Arts and Sciences. He received the B.E. degree in management sciences and engineer from SouthWest Jiaotong University, China. He has worked for Department of Economics and Management, Chongqing University of Arts and Sciences, China as a lecturer since 2010.

Hongjun WANG is a lecturer in Department of Economics and Management, Chongqing University of Arts and Sciences. She received the B.E.and M.E. degree in management sciences and engineer from SouthWest Petroleum University, China. He has worked for Department of Economics and Management, Chongqing University of Arts and Sciences, China as a lecturer since 2006. 